\title{
A Model of Tool Wear in Electrical Discharge Machining Process Based on Electromagnetic Theory
}

Jingyu Pei ${ }^{*}$, , Lenan Zhanga, Jianyi Du, Xiaoshun Zhuang, Zhaowei Zhou, Shunkun Wu, Yetian Zhu 


\title{
A Model of Tool Wear in Electrical Discharge Machining Process Based on Electromagnetic Theory
}

\begin{abstract}
In electrical discharge machining (EDM) process, tool wear is an inevitable phenomenon that adversely affects the geometrical accuracy of machined features. A theoretical model accounting for tool wear during EDM process is hence the basis study for high precision machining. However, in most modeling studies on tool wear and electrode shape, the sparking process is only factorized by the geometric configuration, i.e. the distance between electrodes. The real sparking process related to the fundamental physics is not addressed in these geometric models, which can produce large discrepancies with the experimental results. In this paper, a model of tool wear in EDM is proposed, which accounts for the electric field inside the dielectric fluid using electromagnetic (EM) theory. The spark is proposed to occur at the position where the local electric intensity reaches maximum and exceeds the breakdown strength of the dielectric fluid. This model is shown to provide the physical insight of the real EDM situation, and to give a more accurate prediction of tool wear compared with traditional geometric property based modeling. With these merits, this proposed model can be applied to predict tool wear in various machining processes. To evaluate this model, simulations of EDM die sinking and ED milling are carried out. The results by this electric field model were compared with both geometric model and experiments. By analyzing the profiles of the tool end, the differences in mechanism between the electric field and geometric model are identified. In addition, this electric field model is also applied to simulate the conic tool forming process in the fix-length compensation with micro-milling, which cannot be thoroughly addressed by the geometric model. The model presented in this paper is capable of capturing the key features of the tool wear in a variety of machining processes.
\end{abstract}

Keyword: tool wear, modeling, EDM, electric field, electromagnetic theory

\section{Introduction}

Electrical discharge machining (EDM) is one of the most extensively used non-conventional material removal processes ${ }^{[1]}$. Its unique feature of using thermal energy to machine electrically conductive parts regardless of their hardness has been a distinctive advantage in the manufacturing of mold, die, automotive, aerospace and surgical components, which are difficult to manufacture by conventional machining ${ }^{[1]}$.

The thermoelectric heating process occurring in EDM process erodes the electrode, and thus renders wear ${ }^{[2]}$.The errors caused by the electrode wear result in decreased machining accuracy in the workpiece geometry ${ }^{[3]}$. In the EDM drilling of blind holes, the electrode length is worn, and thus the real depth of the hole is significantly shorter than the target. Moreover, the wear of electrodes adversely affects the geometric precision of deep holes ${ }^{[4]}$. While machining complex 3D micro-cavities, such change becomes more complicating ${ }^{[3]}$. Particularly, in micro-EDM, the machined micro-structures will suffer from tool wear severely, which can eventually produce unacceptable errors ${ }^{[5]}$.

To better understand the tool wear process, many experimental and modeling methods are developed ${ }^{[6-7]}$. Naotake Mohri ${ }^{[2]}$ observed the time dependence of an electrode shape through on-the-machine measurement. Pham et al. ${ }^{[8]}$ conducted a series of die-sinking machining and ED milling experiments to investigate the shape change of electrodes, and their experiments confirmed that the electrode evolves to a stable shape during machining.

Besides the experimental investigations, Z.Y.Yu et al. ${ }^{[4]}$ proposed a wear model of simple-shaped tool using analytical methods. This model could be applied to uniform tool wear conditions. Young et al. ${ }^{[9]}$ developed a two-dimensional geometric model of EDM drilling with cylindrical tools. The model, taking both end and corner wear of tool into consideration, was applied to on-line compensation methods. Segon Heo et al. ${ }^{[10]}$ introduced a three-dimensional geometric simulation method for micro-EDM milling process to predict machined geometry, which could be used to optimize the parameters of actual machining processes and to provide improvements for CNC programs by implementing compensation. Kunieda and Kiyohara ${ }^{[11]}$ developed a geometric simulation for EDM die-sinking inverse process to obtain the appropriate tool electrode shape for retrieving the desired workpiece shape. Several coupled key factors were taken into account, including tool wear, debris particle concentration and tool motion. Zhang et al. ${ }^{[12]}$ developed a two-dimensional geometrical simulation for fix-length milling with cylindrical tools. A clear explanation of the forming mechanism of cone-shaped electrode was provided, and precise predictions of the cone angle were made when layer thickness was less than $85.5 \mu \mathrm{m}$.

Generally, the simulation process includes three steps: (1) searching the discharge point pair; (2) removing materials from the tool and the workpiece; (3) tool feeding. The first step is to search the discharge point pair. Physically, the discharging occurs when the local electric intensity exceeds the breakdown strength of medium. In traditional tool wear modeling, however, spark is commonly located at positions with the shortest gap distance between electrodes, assuming that the electric intensity is 
inversely proportional to scale of gaps. Unfortunately, this assumption is not necessarily true especially when the tool end has large curvatures, in which the corona discharge is dominant. In such cases, geometric prediction based on gap distance failed. Therefore, an electric field model needs to replace the geometry-based model to predict the sparking positions more intrinsically.

The limitations of the geometric model mentioned above was also verified by some recent works. For example, in a recent fix-length compensation milling research by Zhang et al. ${ }^{[12]}$, when the layer thickness was larger than $85.5 \mu \mathrm{m}$, corresponding to a sharper conic angle at the end of electrode, the simulation resulted in large errors compared to the experimental results. Pham's experiment ${ }^{[8]}$ also showed that the electrode shape should remain unchanged with a flat bottom and a worn corner when the drilling depth is greater than $180 \mu \mathrm{m}$. However, the simulation proposed by Zhang et al. ${ }^{[12]}$ based on the shortest gap distance scheme affirmed a semispherical electrode in the end. Therefore, geometry based simulation does not work well when the electrode profile has large and varying curvatures.

To improve the prediction of tool wear, this paper proposes a model applying Gauss's law in Maxwell EM theory and incorporating the electric field between electrodes in the sparking process. The developed modeling tool is proved by experiments with better accuracy in finding the sparking points and predicting tool wear. Taking the fundamentals of physics into the sparking process, this model is thus able to explain and predict tool wear in various EDM processes.

\section{Methodology}

In this paper, an electric field model is presented, where the sparking points are determined based on the electric field distribution in the dielectric fluid. In brief, the sparking occurs at the position where the local electric intensity reaches maximum and exceeds the breakdown strength of the dielectric fluid. In this model, the mesh techniques are similar to what has been proposed by Jeong et al. ${ }^{[9]}$ and Zhang et al. ${ }^{[12]}$ : Both electrodes are meshed into squares of the same size, as shown in Figure 1. The tool travels following an equation of motion determined by the machining parameters. The electrical spark occurs only once in each time step of the simulation. The time scale of each time is indicated in Equation 1.

$$
\Delta t=\frac{1}{f_{\text {spark }}}
$$

where $\Delta t$ is the span of the time step, and $f_{\text {spark }}$ is the maximum spark frequency.

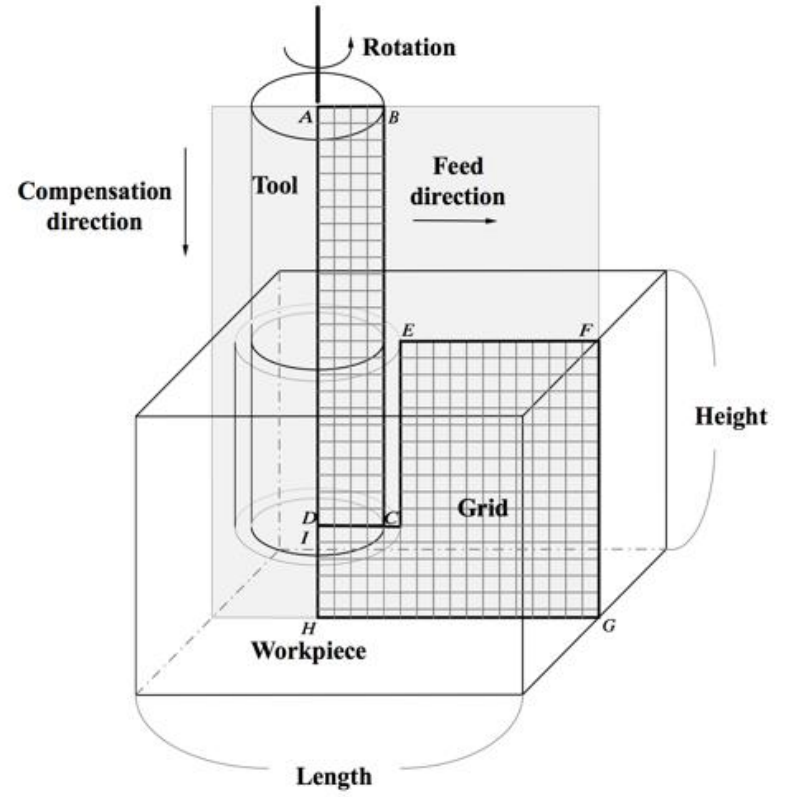

Figure 1 The two-dimensional model of EDM machining process ${ }^{[12]}$. The color of the grids profiles both tool and workpiece. al. $^{[12]}$.

The electric field model in the present study is based on the following eight assumptions. The first five are from Zhang et

(1) The cylindrical electrode remains axially symmetrical during the whole machining process due to rotation;

(2) There is only one spark in each simulation step ${ }^{[7]}$;

(3) The energy delivered by one spark in each simulation step is a constant. That is to say, the removed volume of the material on the electrode is a constant ${ }^{[9,13]}$;

(4) The size of plasma channel is ignored. In other words, the energy delivered by one spark is transferred point to point, rather than area to area ${ }^{[4]}$;

(5) The crater generated by one spark is hemispheric. All the materials in the hemisphere are removed by the dielectric 
fluid;

(6) The electric field reaches its steady state before breakdown;

(7) The initial breakdown of dielectric fluid occurs at the point inside the fluid, where the electric intensity reaches the maximum and exceeds the breakdown strength of the dielectric fluid. The breakdown strength is estimated by voltage difference over sparking gap;

(8) The discharging channel coincides with the electric field line through the sparking point, which ends at the tool and workpiece surface, respectively.

It is worth mentioning that assumption (5) is valid as a trade-off between precision and simplicity. For one thing, several experimental studies have indicated the topological configuration of craters ${ }^{[1,15,16]}$, and there are also researches proposing models and numerical simulations about the shape of a crater using molecular dynamics ${ }^{[17]}$, atomistic-continuum model ${ }^{[18]}$ or heat transfer equation in solid ${ }^{[19]}$. All these models provide good approximations of the crater shape, but as they all depict a single crater only, they are not applicable to machining process simulations. For the other, Zhang's results ${ }^{[12]}$ demonstrate that assumption (5) is a good approximation for the machining process simulation to simplify the computational complexity. Thus, to ensure a calculation efficiency of this model, assumption (5) is held true in the present model.

Based on Gauss law of Maxwell equation, the electric field distribution is depicted by the following equation,

$$
\nabla \cdot \boldsymbol{E}=\frac{\rho}{\varepsilon_{0} \varepsilon}
$$

where $\boldsymbol{E}$ is the electric field, $\rho$ is the electric discharge density, $\epsilon_{0}$ is the permittivity of free space, and $\epsilon$ is the relative permittivity of work fluid.

Assumption (6) of steady state for electric field implies that Equation (2) can fully express the electric field distribution in the fluid before breakdown. Since there are no charges inside, Equation (2) can be simplified to the Laplace equation and be described in the Cartesian coordinates as

where $U$ is the potential of electric field.

$$
\nabla^{2} U=0
$$

The boundary conditions implemented in this model are depicted in Figure 2. The boundaries of tool (boundary 1, 2 and 3 respectively in Figure 2) have the potential of $200 \mathrm{~V}$, whereas the potential of the workpiece (boundary 6) is assumed to be zero. Physically, the potential of the dielectric fluid at infinity (boundary 4, 5, 7 and 8 respectively in Figure 2) should also be zero. However, in the numerical simulation, it is impractical to have an infinitely large domain. Thus, it is reasonable to assume that the potential becomes zero at points far enough from the electrodes.

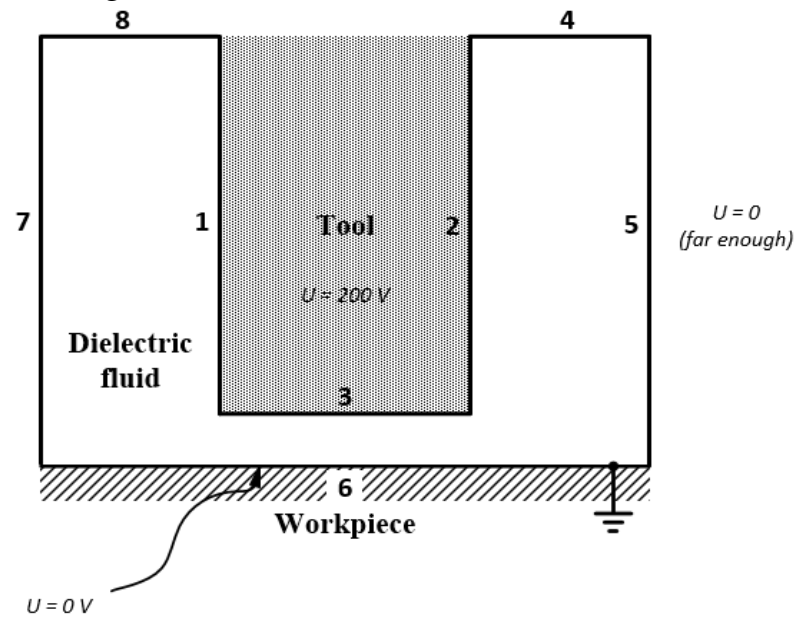

Figure 2 Boundary conditions of the potential distribution in the electric field model: The distribution is depicted within the dielectric fluid, which adjoins the tool along boundary 1,2 and 3. Boundary 6 separates the dielectric fluid and the workpiece. Boundary 4, 5, 7 and 8 are assumed at infinity.

At the beginning of each time step, the electric field is redistributed and must be re-calculated by solving Equation (3) using finite difference method. The electric intensity at all points inside the dielectric fluid can then be evaluated. If the maximum value exceeds the threshold intensity set as the breakdown voltage of the fluid, sparking originates in accordance with assumption (7). If there are more than one points with the same maximum intensity, one is randomly chosen. When sparking commences, the discharge will spread along an immediately formed channel to the ends of both tool and workpiece surfaces, and such discharging channel is found by applying assumption (8). At both ends of the discharging channel, the removal process occurs, resulting in new profiles of the electrodes. If the maximum electric intensity does not exceed the breakdown intensity, the feeding process will be initiated to update the tool's position. Such processes are repeated in every time step $\Delta t$. In Figure 3, a flowchart of the simulation processes is shown for clearer illustrations. 


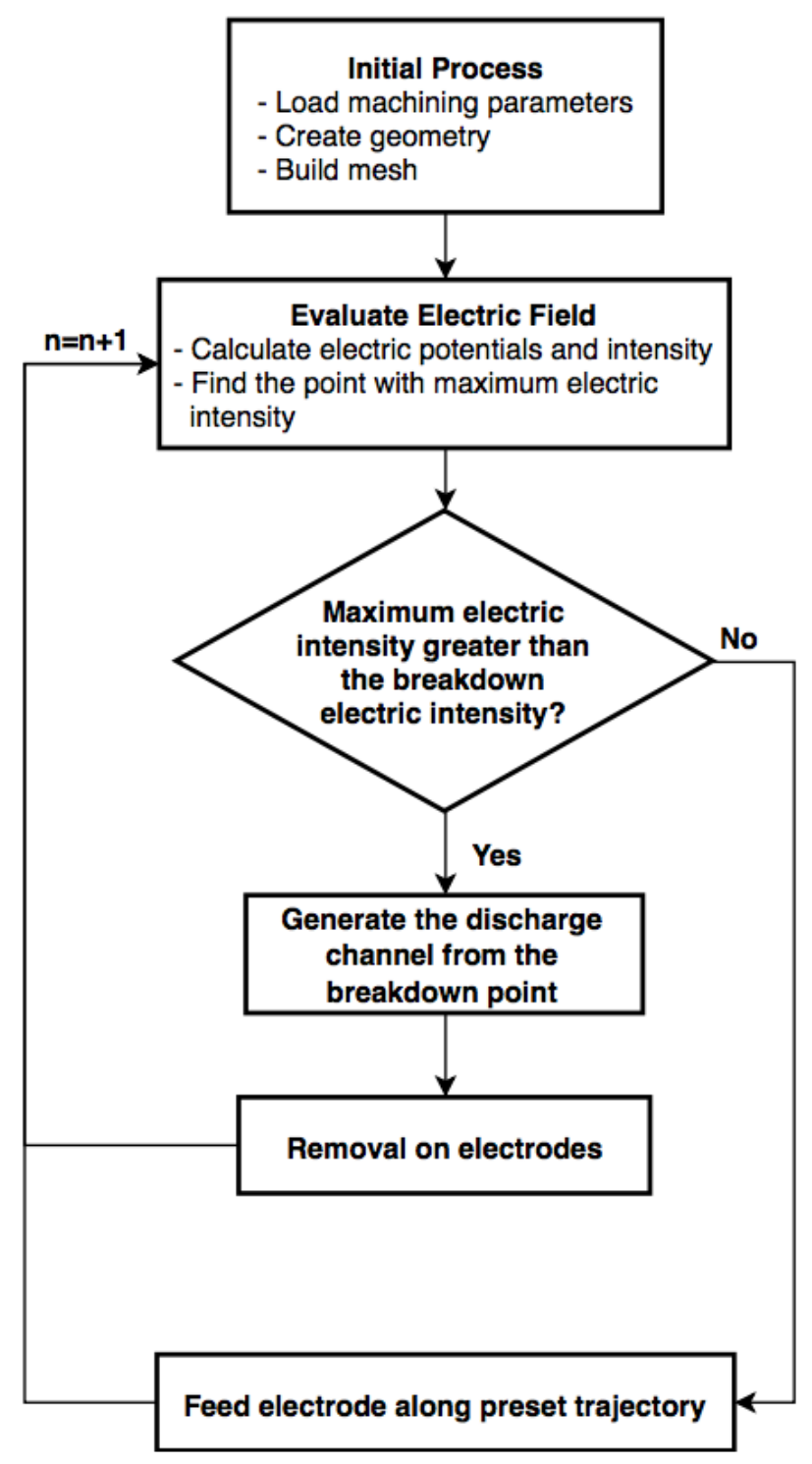

Figure 3 Flow chart of the EDM machining model based on electric field simulation.

The removal criterion is determined by assumption (5). The crater radius is calculated by Equation (4),

$$
r_{t}=r_{w} \theta^{\frac{1}{3}}
$$

where $r_{t}$ and $r_{w}$ are the radius of the crater on tool and workpiece respectively, and $\theta$ is the volumetric relative wear ratio related to the materials of the electrodes.

\section{Validation and Application}

In this section, the proposed electric field model is validated by comparing the results with those from the geometric model and the experiments. To achieve this, EDM processes with different servo motions and electrode shapes are applied in the following aspects:

First, the die sinking process with only axial electrode motion is chosen due to its wide applications in mould machining. In this scenario, various electrode shapes are studied from simple to complex, and the results produced by the proposed model are compared with those retrieved from the geometric model and the experiments. Then, this electric field model is further applied in fix-length compensation milling to simulate the conic tool forming process.

\subsection{Die-sinking process simulation and experimental verification}

Die-sinking processes with different electrode shapes are simulated in both the geometric and electric field models. To 
begin with, the tool wear of the conic tool is studied and then compared with the experimental results. A series of simulations using wedge-shaped tools are followed to better discuss and analyze the key differences found from two models. At last, a comprehensive simulation with a dual-tip tool clearly visualizes and verifies the different model mechanisms indicated in previous scenarios.

\subsubsection{Simulations and experiments set-up}

In order to validate the electric field model as outlined previously, its simulation results are compared with those obtained by the geometric model and the experiments respectively. To clearly identify the differences between both models, a conic tool is used in the simulation, as shown in Table 1. The simulation parameters are listed in Table 2. The diameter of the electrodes is chosen to be $200 \mu \mathrm{m}$.

Table 1 Geometric configurations of tools used in die sinking simulation.

\begin{tabular}{|c|c|c|c|c|c|}
\hline Tool name & Conic tool & $\begin{array}{l}\text { Wedge-shaped } \\
\text { tool } 1\end{array}$ & $\begin{array}{l}\text { Wedge-shaped } \\
\text { tool } 2\end{array}$ & $\begin{array}{l}\text { Wedge-shaped } \\
\text { tool } 3\end{array}$ & Dual-tip tool \\
\hline \multicolumn{6}{|l|}{ Schematic drawing } \\
\hline Diameter/width $[\mu \mathbf{m}]$ & 200 & 200 & 200 & 200 & 200 \\
\hline \multirow[t]{2}{*}{ Tip angle } & 90 degrees & Left: 45 degrees & Left: 60 degrees & Left: 60 degrees & Left: an arc with \\
\hline & & $\begin{array}{ll}\text { Right: } & 135 \\
\text { degrees } & \end{array}$ & $\begin{array}{ll}\text { Right: } & 120 \\
\text { degrees } & \end{array}$ & $\begin{array}{ll}\text { Right: } & 120 \\
\text { degrees } & \end{array}$ & $\begin{array}{l}\text { a central angle of } \\
120 \text { degrees } \\
\text { Right: an } \\
\text { isosceles triangle } \\
\text { with base angle } \\
\text { of } 30 \text { degrees }\end{array}$ \\
\hline
\end{tabular}

Table 2 Simulation parameters for die-sinking process.

\begin{tabular}{lll}
\hline Item & Unit & Value \\
\hline Grid size & $\mu \mathrm{m}$ & 0.5 \\
Crater diameter on workpiece & $\mu \mathrm{m}$ & 5 \\
Simulation time step & $\mu \mathrm{s}$ & 56.4 \\
Tool wear ratio & - & $10.0 \%$ \\
Breakdown intensity & $\mathrm{V} / \mu \mathrm{m}$ & 25 \\
\hline
\end{tabular}

The experiments were conducted on Charmilles Technologies ROBOFORM $35 * 2400$. Electrodes were fixed with the chuck. Table 3 lists the machining parameters. The machining process was paused at different machining depths, and the corresponding electrode shapes were captured with microscope (503+ GAOSUO digital microscope) for comparisons. In the experiments, it is difficult to shape such small electrodes following the dimensions in Table 1. Therefore, real electrodes are reasonably scaled up and made planar with a thickness of $1 \mathrm{~mm}$, while the cross section shapes follow those in Table 1 . 
Table 3 Machining parameters for die-sinking process.

\begin{tabular}{lll}
\hline Item & Unit & Value \\
\hline Tool material & - & Copper \\
Workpiece material & - & Mirrax die steel \\
Dielectric fluid & - & $\begin{array}{l}\text { Kerosene-based } \\
\text { mixture }\end{array}$ \\
Tool width & $\mathrm{mm}$ & 3 \\
Dual-tip tool width & $\mathrm{mm}$ & 6 \\
Tool polarity & - & + \\
Open circuit voltage & $\mathrm{V}(\mathrm{DC})$ & 200 \\
Peak gap current & $\mathrm{A}$ & 2 \\
Sparking on time & $\mu \mathrm{s}$ & 50 \\
Sparking off time & $\mu \mathrm{s}$ & 6.4 \\
Flushing & - & $\mathrm{Off}$ \\
\hline
\end{tabular}

Although the electrode sizes in experiments and simulations are different because of the stated machining limitations, both results are still comparable. As the main difference between micro- and macro-EDM is the volumetric relative wear ratio, one can affirm that the scale has negligible effects on the final shape of the electrode. The macroscopic experiments are thus able to indicate the geometry in the micro-EDM simulation. Besides, in order to avoid the interference caused by secondary discharge of deep-hole machining, no flushing condition is applied to increase the volumetric relative wear ratio thereby decreasing the machining depth.

\subsubsection{Results from simulation and experiment with conic tool}

As mentioned in Section 2, the electric field is re-calculated in every time step. As an example, Figure 4 shows the electric potential distribution before machining for a conic tip electrode. In Figure 4, the potential gradient at the conic tip reaches the maximum in the dielectric fluid, indicating the largest field intensity. In the following context, the probability to spark in the region near the conic tip is demonstrated to be maximized. Such phenomenon is further validated in the real machining process, where material close to the electrode tip tends to be worn first. Therefore, the potential distribution in Figure 4 physically accords with the previous understandings of EDM.

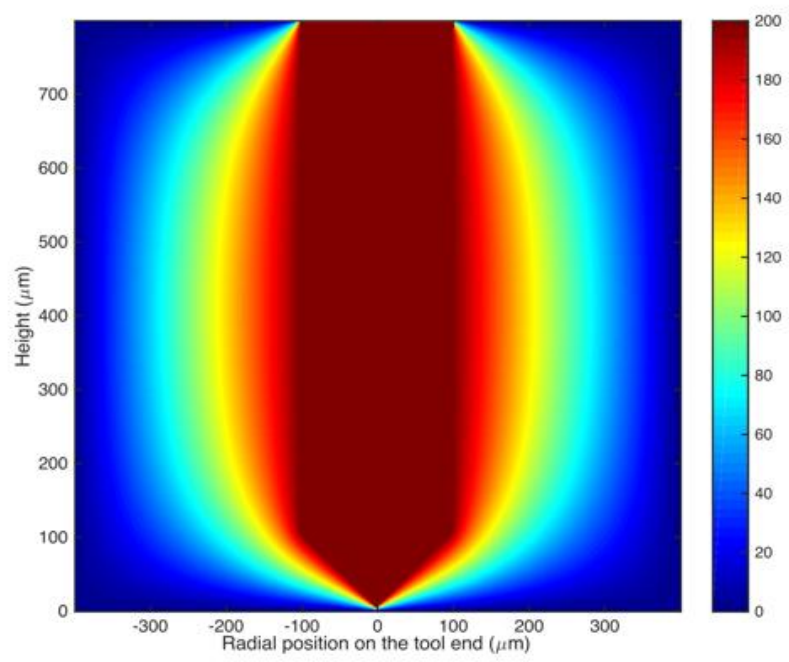

Figure 4 Contour of the potential distribution in the dielectric fluid.

Figures 5 and 6 respectively show the simulation results of the geometric model and the proposed electric field model. One can observe that the original conic tips remain conic in Figure 5 but become gradually flat in Figure 6, indicating larger wear at the tips in the electric field model. This feature demonstrates that spark is more frequent at the point with a larger curvature, in accordance with the corona discharge phenomenon. Compared with tool wear at the equivalent drilling depth in the experiments shown in Figure 7, the electric field model produces more consistent electrode shapes with the experiments than the geometric model. Furthermore, according to the regularity shown in Figure 6 and Figure 7, we can predict that the conic tip will be totally worn off as the depth goes large enough. 


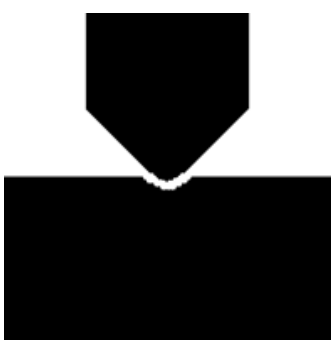

(a)

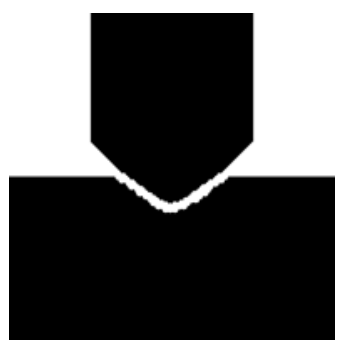

(b)

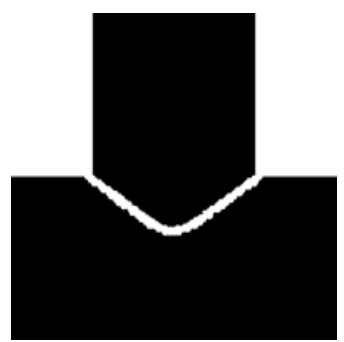

(c)

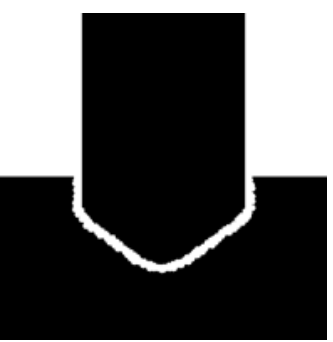

(d)

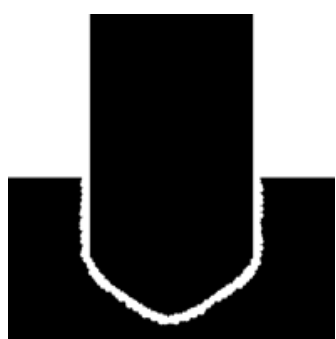

(e)

Figure 5 Change of 45-degree tool shape in geometric model with different depths. (a) $16 \mu m$; (b) $44 \mu m$; (c) $73 \mu m$; (d) $118 \mu m$; (e) $179 \mu m$. The tool remains the unchanged shape while it is sinking, corresponding to spatially-even sparking along the surface.

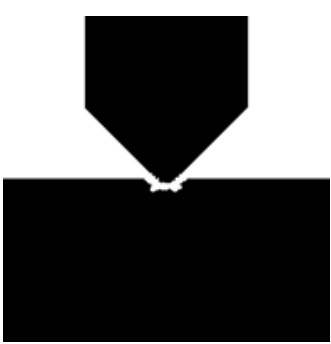

(a)

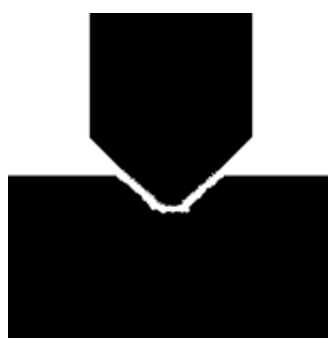

(b)

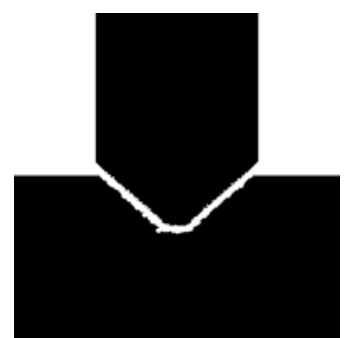

(c)

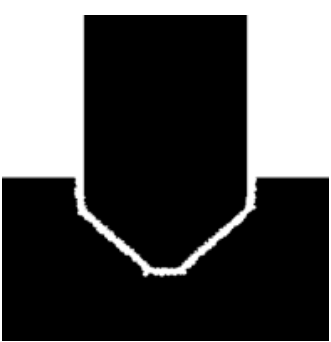

(d)

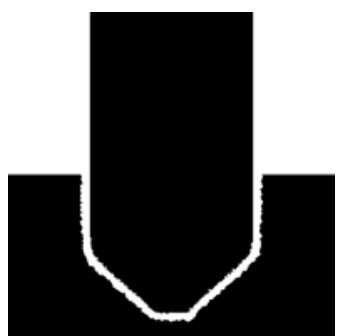

(e)

Figure 6 Change of 45-degree tool shape in electric model with different depths. (a) $16 \mu m$; (b) $44 \mu m$; (c) $73 \mu m$; (d) $118 \mu m$; (e) $179 \mu m$. The tool tip is being greatly worn while it is sinking, corresponding preferred sparking at the tip.

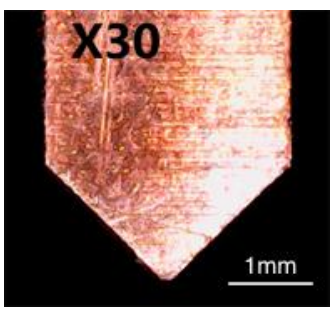

(a)

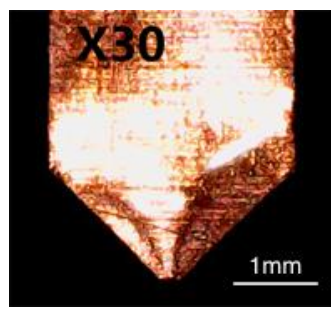

(b)

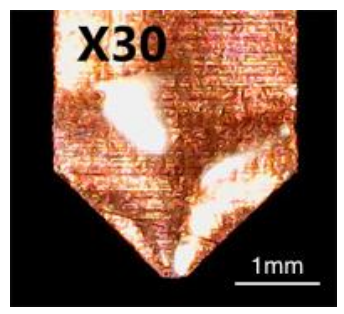

(c)

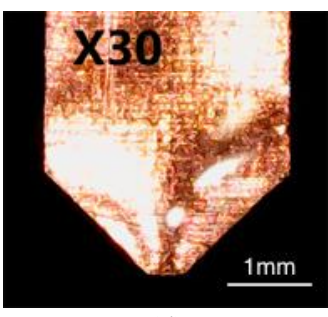

(d)

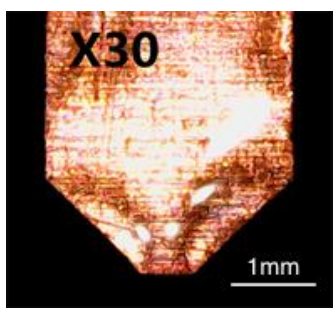

(e)

Figure 7 Change of 45 -degree tool shape in experiment with different depths. (a) $20 \mu m$; (b) $57 \mu m$; (c) $90 \mu m$; (d) $142 \mu m$; (e) $220 \mu m$. The experimental results show accordance to the electrical field model results with a flat tip.

\subsubsection{Analysis of the differences between models based on electric field and geometry using wedge tools}

A wedge-like tool with different angles, as shown in Table 1, is applied in the simulations to further look into the discharging mechanisms due to different curvatures. The tool is inserted in the workpiece at the beginning of the simulation, also depicted in Table 1. Simulation parameters of the wedge tools are also found from Table 2.

The shapes of the tools simulated by the two models are depicted in Figure 8 and Figure 9. As is clear from Figure 9 of the electric field model, the tips of the tool are worn flat. After the tip is truncated, the tool end keeps flat all the way down. From the results of the geometric model shown in Figure 8, however, the tool is worn in such a way that the sparking positions are generally uniformly distributed along the surface with both ends slightly worn off more. Hence, this model renders a smoother end profile.

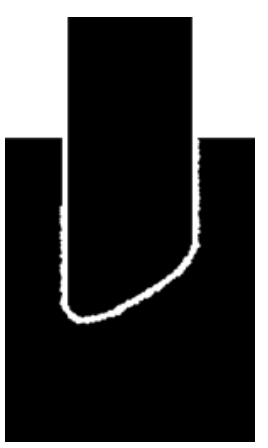

(a)

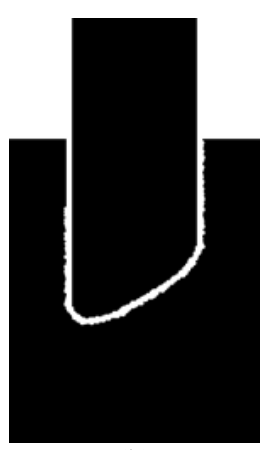

(b)

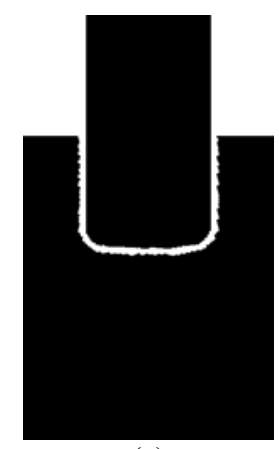

(c)

Figure 8 Stable shapes of tool and workpiece for the geometric model. (a) Tool 1, with 45 degrees; (b) Tool 2, with 60 degrees; (c) Tool 3, with 90 
degrees. There is a smooth wear distribution along the tool surface.

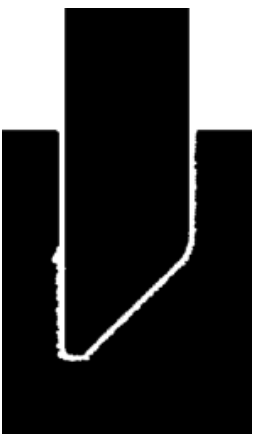

(a)

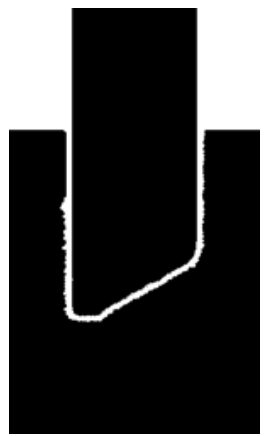

(b)

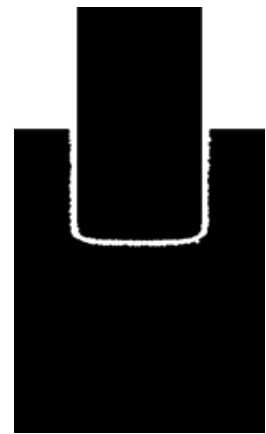

(c)

Figure 9 Stable shapes of tool and workpiece for the electric field model. (a) Tool 1, with 45 degrees; (b) Tool 2, with 60 degrees; (c) Tool 3, with 90 degrees. The tip of all the tools are significantly worn out, while the other part of the tool surface keeps the original shape.

To quantitatively measure the impact of sparking on the tool shape, one can visualize the tool wear by plotting the radial sparking distribution along the tool surface. In this paper, the sparking frequency is defined as the ratio of local sparking counts at each position to the total sparking counts. Physically, the point with higher sparking frequency renders larger wear, showing that the sparking distribution is able to quantify the tool wear.

Figure 10 shows the sparking frequency distribution along the tool surface retrieved by both models. The $\mathrm{x}$ - and $\mathrm{y}$-axises represent the radial position from the symmetric axis at the end of the tool, and the sparking frequency varying with the radial position. In each subfigure, three curves are given, corresponding to tools with left tip angles of 45, 60 and 90 degrees respectively.

In both the geometric and the electric models, as shown in Figure 10, sparking frequencies on both ends are relatively higher, corresponding to larger tool wear. One can refer to the simulation results in Figure 8 and 9, and the sparking frequency distributions are in good accordance with tool wear.

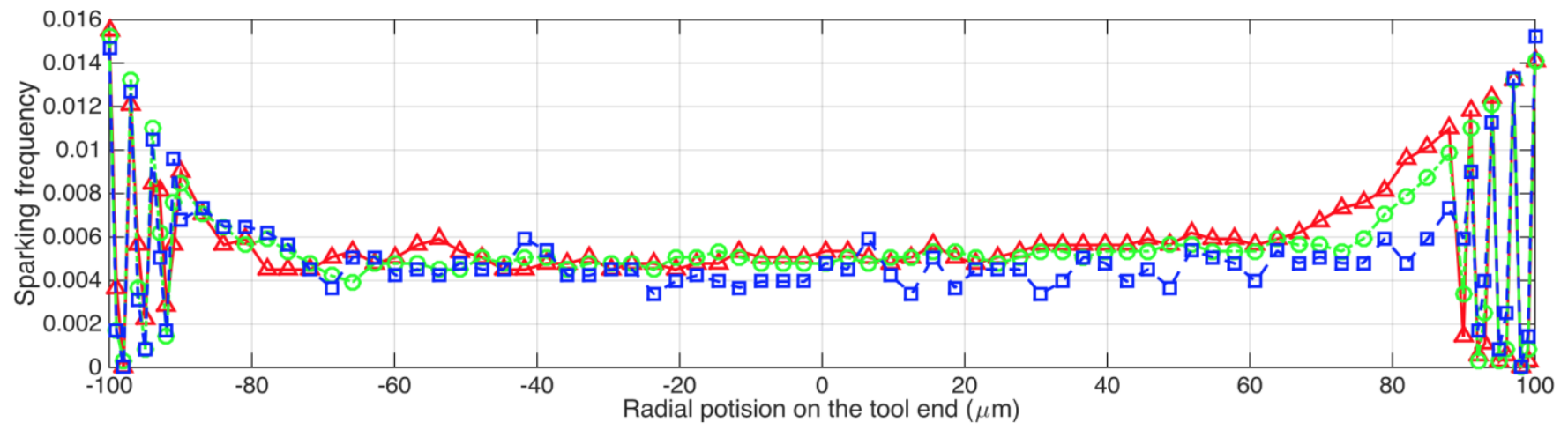

(a) Geometric model results;

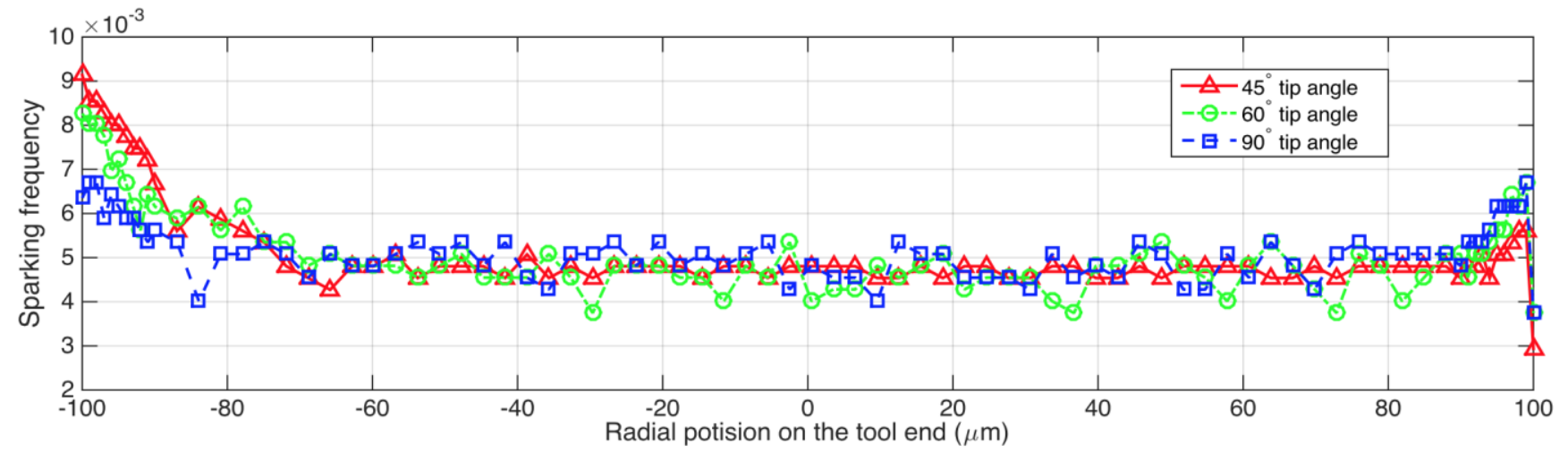

(b) Electric field model results.

Figure 10 Sparking frequency for tool with different tip angles. Symmetric and overlapped frequency curves shown in the results of geometric model, 
whereas asymmetric frequency curves starting from different positions depicted in the results of electric field model.

As Figure 10 shows, the sparking frequency is U-shaped, in which larger tool wear occurs on both ends. The sparking frequency curve for the geometric model shown in Figure 10(a) is symmetric with respect to the tool axis. Besides, all three curves with different tip angles nearly overlap with each other. Both observations indicate that results from the geometric model are independent of the tip angle and the curvatures of tools. In contrast, frequency curves for the electric model in Figure 10(b) show clear asymmetry, as well as difference in frequency on both ends with different tip angles. As an example, the red curve, representing the tool with a left tip angle of 45 degrees, starts with the maximum sparking frequency of 0.009 at $-100 \mu \mathrm{m}$ and ends with the minimum frequency of 0.003 at the right tip of 135 degrees. Figure 10(b) hence demonstrates that in the electric model, results are changing with tip angle and surface curvature in two aspects: three curves start and end with different frequencies rather than overlap with each other; none of the curves is symmetric because of the geometric asymmetry.

Figure 11 summarizes the sparking frequency changing with five tip angles (45, 60, 90, 120 and 135 degrees) from the two models, clearly showing the independency of tip angle in the geometric model as the sparking frequency remains constant at around 0.015. However, in the electric field model, sparking frequency decreases significantly with the increase of the tip angle.

Because of the electric field model's stronger dependence on the curvature of the tip than the geometric model, it is a more accurate reflection of the real EDM situation, where points with larger curvatures on the electrode wear more quickly. As shown in Figure 11, a sharper tip with a larger curvature leads to more frequent sparking, thus rendering larger wear. It can be concluded that the electric field model is better at predicting the electrode wear than the geometric model.

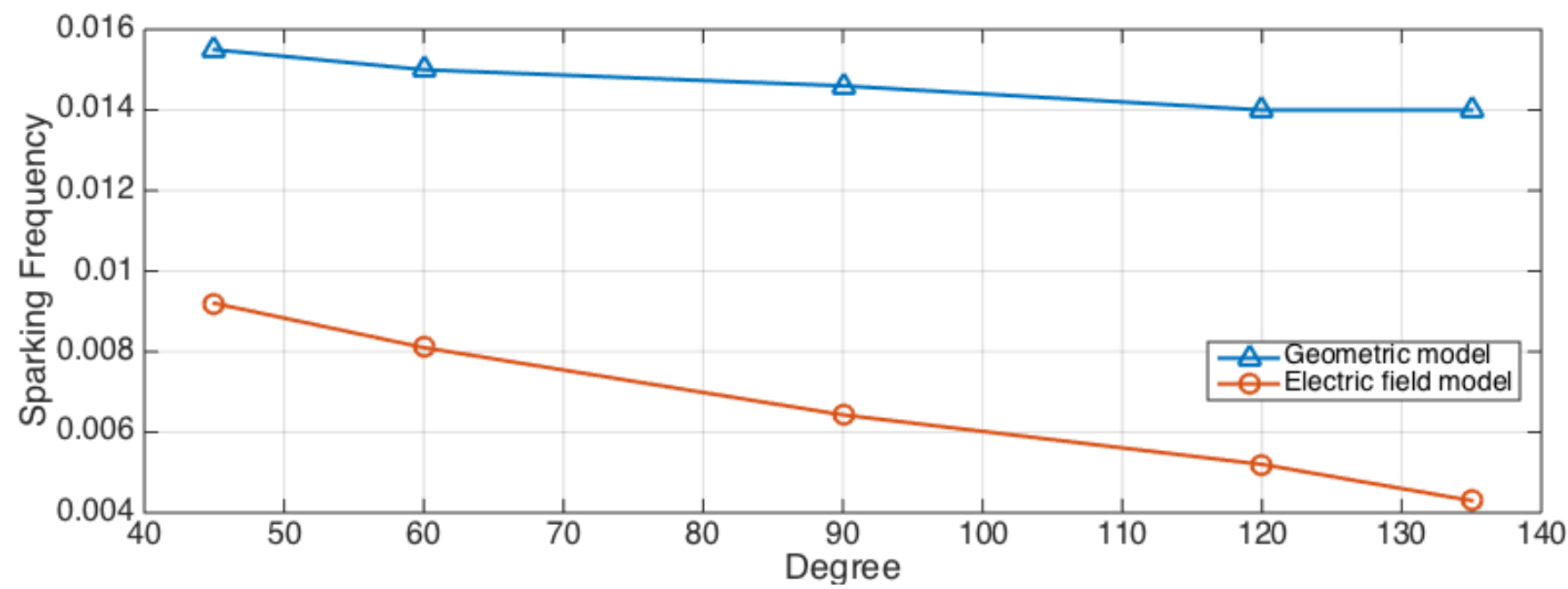

Figure 11 Sparking frequency at tool tips varying with tip angles. Different response sensitivity to tip angle revealed by geometric and electric field model respectively.

The difference between the geometric and the electric field model in the tool wear and sparking frequency originates from the difference between the mechanisms of two models. Compared with the electric field model presented in this paper, the sparking process in the geometric model is based on two criteria, namely, the gap distance between pair of points and the density of edges in a region with the same scale as an erosion crater. In the geometric model, sparking will occur between the pair of points with the shortest distance, thus producing the flat end surface in drilling. If the distance between the tool and workpiece remains constant, the sparking probability at any positions on either side of the tool should be the same, and accordingly, the tool wear in this condition should also be uniform. However, at the corner of the tool, as shown in Figure 19(a), there are circles 1 and 2 enclosing two edges, but in circle 3 on the end surface, the number is only one. As the sparking probability on each edge is equal, the sparking probability in circle 1 and 2 should approximately twice in circle 3 . In the geometric model, the probability of electrode wear at any points is thus proportional to the density of edges. As tips of the three tools with different tip angles in the simulation all have two edges even though they have different angles, they should have the same probability of wear according to the geometric model. However, a key factor missing in the geometric model is the curvature, which is acting as a dominant factor in the electric field model.

\subsubsection{Validation of the electric field model using dual-tip tool}

To validate the hypothesized mechanisms of the electric field model, another simulation was conducted using a specially shaped dual-tip tool shown in Table 1. The tool is composed of an apex and an arc, aligned horizontally. The simulation parameters and the corresponding machining parameters are listed in Table 2.

The sparking frequency simulated by the electric field and the geometric model are shown in Figure 12. The left and right 
peak of the frequency curve represent the sparking frequency of the left arc and the right apex tip, respectively.

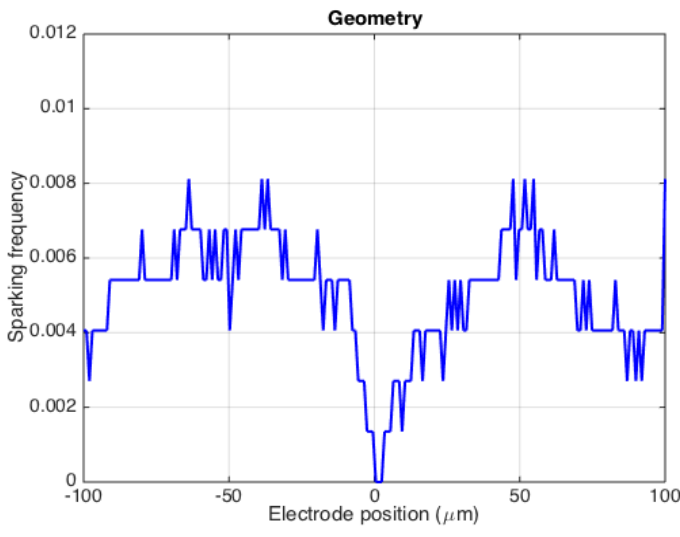

(a)

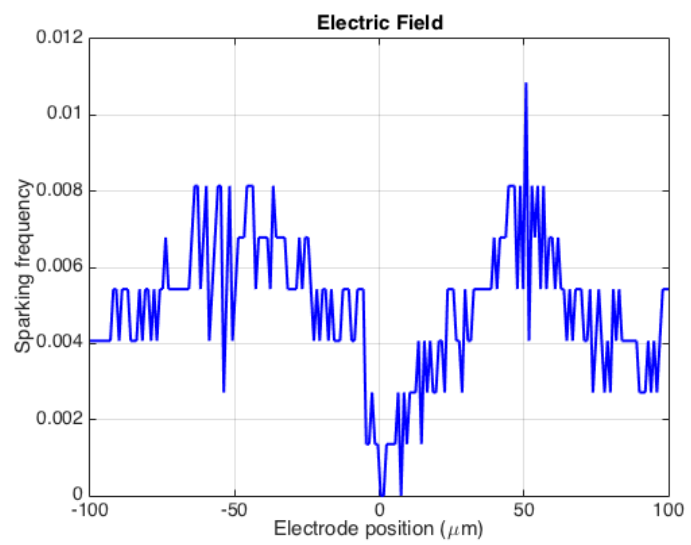

(b)

Figure 12 Sparking frequency along the dual-tip tool's end. (a) Geometric model results; (b) Electric field model results. Sparking frequency is dramatically large at left apex in electric field model.

As seen from the results of geometric model in Figure 12(a), the magnitude of the left and right peak are really close, indicating independence on the curvature of tips, thus verifying that the density of edges is the dominant factor in the geometric model. On the other hand, in Figure 12(b), the sparking frequency at the apex simulated by the electric field model is much higher than its arc counterpart, showing that larger curvatures on the surface increase the probability of sparking.

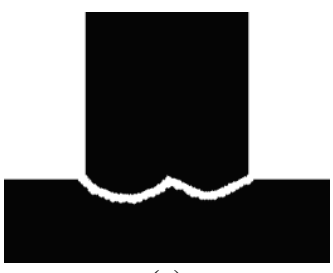

(a)

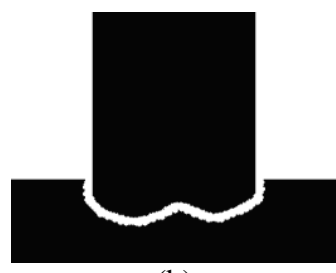

(b)

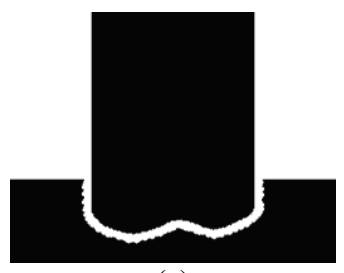

(c)

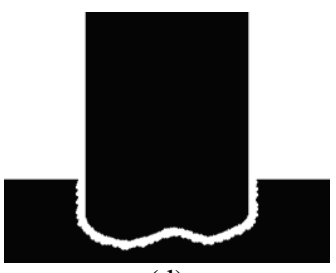

(d)

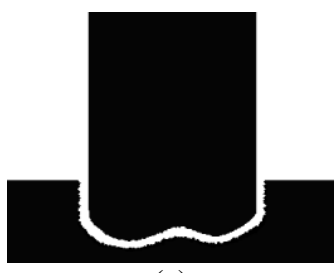

(e)

Figure 13 Change of the dual-tip tool ends geometric model with different depths. (a) $33 \mu \mathrm{m}$; (b) $66 \mu \mathrm{m}$; (c) $90 \mu \mathrm{m}$; (d) $100 \mu \mathrm{m}$; (e) $1000 \mu \mathrm{m}$. The shape of tool remains stable.

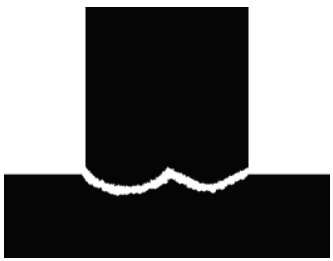

(a)

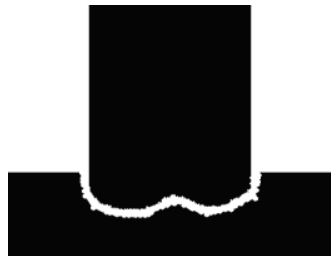

(b)

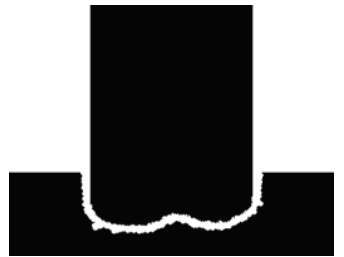

(c)

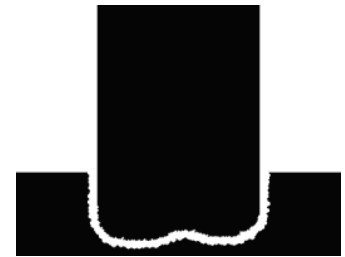

(d)

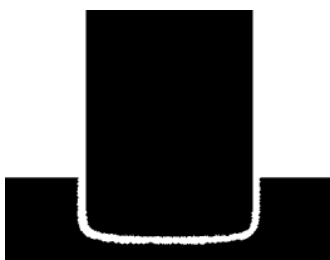

(e)

Figure 14 Change of the dual-tip tools end in electric field model with different depths. (a) $33 \mu \mathrm{m}$; (b) $66 \mu \mathrm{m}$; (c) $90 \mu \mathrm{m}$; (d) $100 \mu \mathrm{m}$; (e) $1000 \mu \mathrm{m}$. Tool end gets plain gradually.

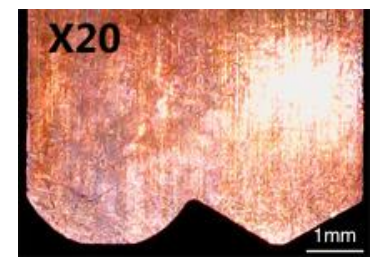

(a)

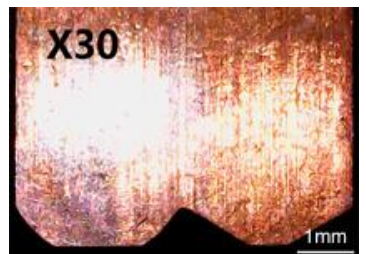

(b)

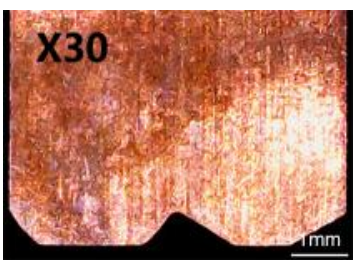

(c)

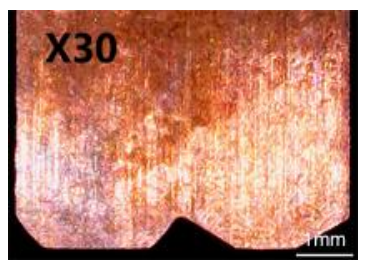

(d)

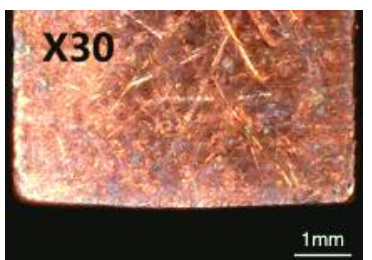

(e)

Figure 15 Change of the dual-tip tool's end in experiment with different depths: (a) $57 \mu \mathrm{m}$; (b) $123 \mu \mathrm{m}$; (c) $178 \mu \mathrm{m}$; (d) $200 \mu \mathrm{m}$; (e) $1000 \mu \mathrm{m}$. Results qualitatively agree with electric field model.

Figure 13 to 15 shows the shapes of the tool at varying depths retrieved from both models and the experiments. From Figure 13(a) to (e), the shape of the tool remains stable due to the nearly uniform tool wear, indicating as previously that a tip with larger curvature does not lead to larger wear in the geometric model. However, in the electric field model in Figure 14, both tool ends become flat gradually as the drilling depth increases, suggesting a relatively larger wear at the apex tip of tool. This was later verified by the experimental results presented in Figure 15. Especially, it is of great interest to compare Figure 13(e) with Figure 14(e). When the drilling depth becomes large enough, the shape of the tool end in Figure 13(e) remains 
dual-tip, indicating the steady-state tool profile in the geometric simulation. This is significantly different from Figure 14(e): at the same depth, the tool end predicted by the electric field model becomes flat with smooth corners, which corresponds to the experimental results in Figure 15(e). The discrepancy in Figure 13(e) and Figure 14(e) demonstrates that the geometric model fails in predicting the steady-state tool shape when the tool has varying curvatures.

\subsection{Application of the electric model to the fixed-length compensation in milling process}

In order to examine the extensive applications of the electric field model, the EDM with more complicating two-axis motions should be discussed. A simulation of the ED milling with fixed-length compensation method is carried out to further validate the proposed model in a process integrating rotation, lateral feeding as well as axial compensation of the tool.

Before performing the simulation, it is necessary to give a brief outline of the fixed-length compensation milling technology in EDM. Fixed-length compensation method was first proposed by Pei et al. ${ }^{[20]}$ for micro-EDM milling. This manufacturing strategy is generally characterized by three key parameters, i.e. the layer thickness $H_{\text {comp }}$, the lateral feeding length $L_{c o m p}$ and the compensation accuracy $l_{\text {comp }}$ as shown in Figure 16 . According to Pei et al. ${ }^{[20]}$ and Zhang et al. ${ }^{[12]}$, the spinning tool is supposed to compensate downwards for a fixed length $l_{\text {comp }}$ every time it feeds horizontally by a specific milling length $L_{\text {comp }}$ (see Figure 16). Compared with traditional linear compensation method, the amount of compensation in fixed-length compensation is predetermined by the relative volumetric wear ratio. The time spent on measuring the compensation and dynamically resetting the machining parameters is thus saved. In practice, as the lateral feeding length $L_{c o m p}$ is set to as small as tens of micrometers, the compensation accuracy $l_{\text {comp }}$, which determines the machining accuracy, can be controlled within $1 \mu \mathrm{m}$. As a result, this fix-length compensation strategy can reach higher machining efficiency and accuracy than the traditional linear compensation method.

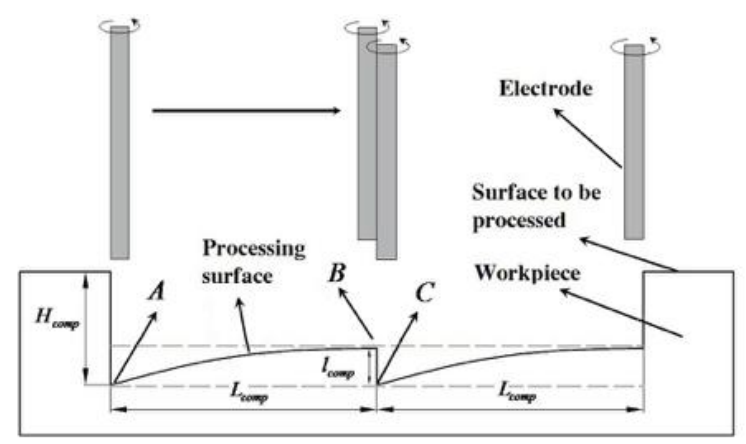

Figure 16 Schematic of the fixed-length compensation theory ${ }^{[12]}$.

As reported by Zhang et al. ${ }^{[12]}$, a conic tool end is inevitable in the fix-length compensation milling. Briefly, the combination of the rotation, lateral feeding and the axial compensation all contribute to the formation of the conic tool end. Such observed conic shape has been interpreted by Zhang et al. ${ }^{[12]}$ in detail. Previous work has shown that it is quite important but challenging to predict the conic angle and the overall profile of tool especially for the layer thickness $H_{\text {comp }}$ larger than 80 $\mu \mathrm{m}^{[12]}$, when the corona discharging effect becomes significant but is not accounted for in the geometric model. However, in following sections, the difficulties outlined above can be fully addressed by the electric field model presented in this paper.

The electric field model is further evaluated by performing a simulation on the fix-length compensation ED milling. The profile of the tool at different stages during the milling process is predicted and compared with experiments. The simulation parameters applied in this work are listed Table 4, which accord with the machining parameters shown in Table 5.

Table 4 Simulation parameters for fix-length compensation milling simulation.

\begin{tabular}{lll}
\hline Item & Unit & Value \\
\hline Grid size & $\mu \mathrm{m}$ & 0.5 \\
Tool diameter & $\mu \mathrm{m}$ & 200 \\
Crater diameter on workpiece & $\mu \mathrm{m}$ & 3 \\
Simulation time step & $\mu \mathrm{s}$ & 8 \\
Tool wear ratio & - & $11.9 \%$ \\
Breakdown intensity & $\mathrm{V} / \mu \mathrm{m}$ & 20 \\
Length of compensation & $\mu \mathrm{m}$ & 60 \\
Compensation accuracy & $\mu \mathrm{m}$ & 1 \\
\hline
\end{tabular}




\begin{tabular}{ll}
\hline Tool feed rate & $\mu \mathrm{m} / \mathrm{s}$ \\
\hline
\end{tabular}

Table 5 Machining parameters for fix-length compensation milling simulation.

\begin{tabular}{|c|c|c|}
\hline Item & Unit & Value \\
\hline Tool material & - & Copper \\
\hline Workpiece material & - & $\begin{array}{l}1 \mathrm{Cr} 18 \mathrm{Ni} 9 \mathrm{Ti} \\
\text { stainless steel }\end{array}$ \\
\hline Dielectric fluid & - & $\begin{array}{l}\text { Kerosene-based } \\
\text { mixture }\end{array}$ \\
\hline Tool diameter & $\mu \mathrm{m}$ & 200 \\
\hline Tool polarity & - & - \\
\hline Open circuit voltage & $\mathrm{V}(\mathrm{DC})$ & 200 \\
\hline Peak gap current & $\mathrm{A}$ & 0.5 \\
\hline Sparking on time & $\mu \mathrm{s}$ & 1.6 \\
\hline Sparking off time & $\mu \mathrm{s}$ & 6.4 \\
\hline Too wear ratio & - & $11.9 \%$ \\
\hline Flushing & - & On \\
\hline
\end{tabular}

Figure 17 shows the tool profiles at different milling length varying from $150 \mu \mathrm{m}$ to $1100 \mu \mathrm{m}$. Accordingly, the tool profiles at the same milling length shown in Figure 18 are obtained from the experiment. Figures 17 (a), (b) and (c) show the initial stage of the milling at which the conic angle cannot be observed yet. Comparing with corresponding experimental results shown in Figures 18 (a), (b) and (c), the simulated tool ends in the initial stage are more flat. One possible reason is the coaxiality of the tool. In the simulation, the tool is assumed to be perfectly axisymmetric in Figure 19(a). But in the experiments, it is not necessarily true, which suggests the spinning of the tool can be affected by coaxiality. Illustrated in Figure 19(b), if the tool spins with lower coaxiality, the apex of the cone will interact with a larger area shown as area B'-B" of the workpiece, and thus the wear around the apex in reality can be larger than the simulations, causing the "steeper" surface on both sides of the tool tip in Figures 18(a), (b) and (c).

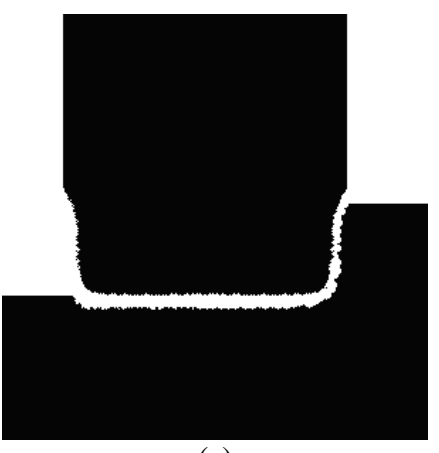

(a)

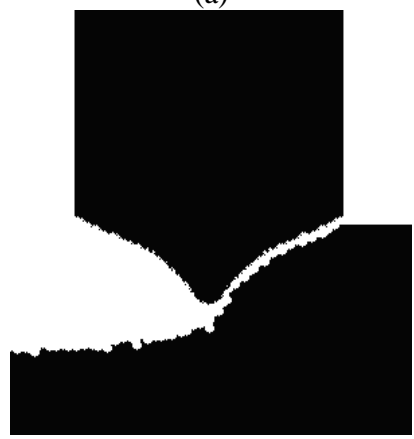

(e)

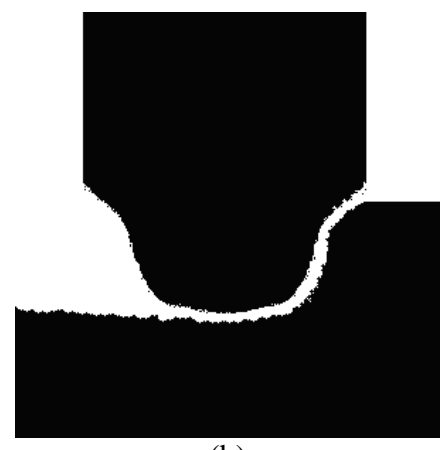

(b)

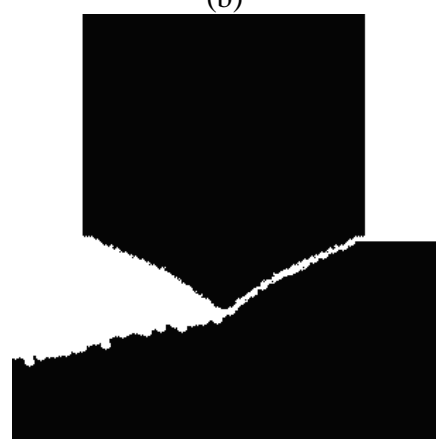

(f)

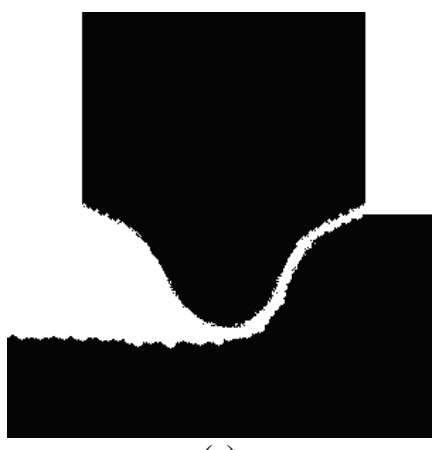

(c)

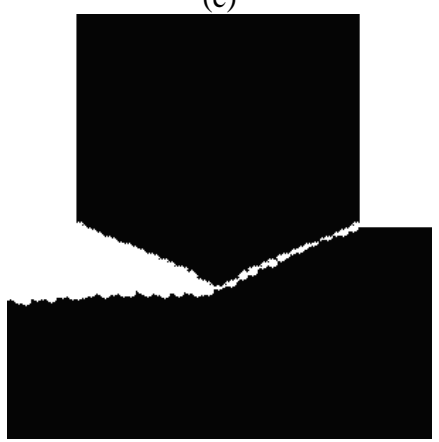

(g)

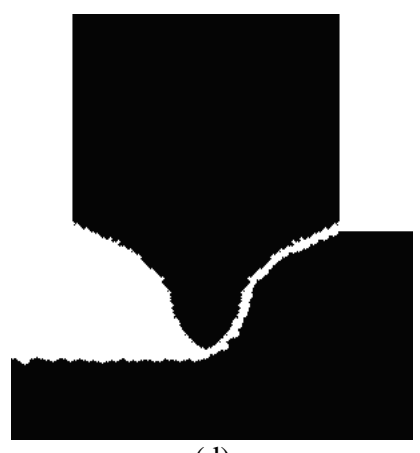

(d)

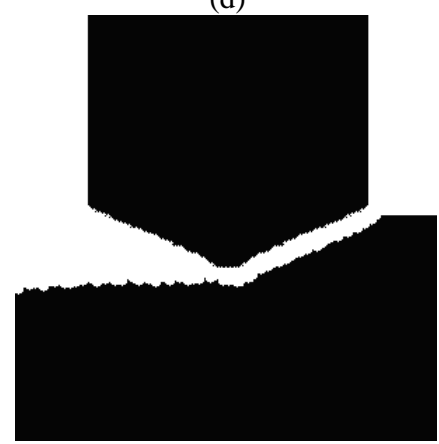

(h)

Figure 17 Change of tools shape in simulation of fixed-length compensation using electric field model with different milling lengths. (a) milling $150 \mu \mathrm{m}$; (b) milling $350 \mu \mathrm{m}$; (c) milling $550 \mu \mathrm{m}$; (d) milling $650 \mu \mathrm{m}$; (e) milling $800 \mu \mathrm{m}$; (f) milling $900 \mu \mathrm{m}$; (g) milling $1050 \mu \mathrm{m}$; (h) milling $1100 \mu \mathrm{m}$. 


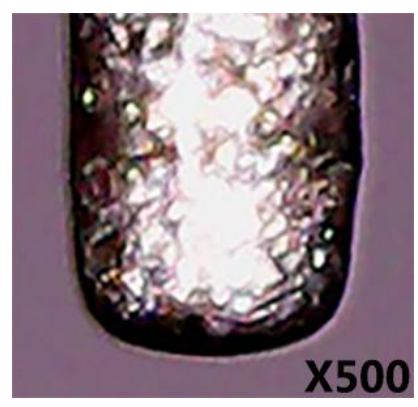

(a)

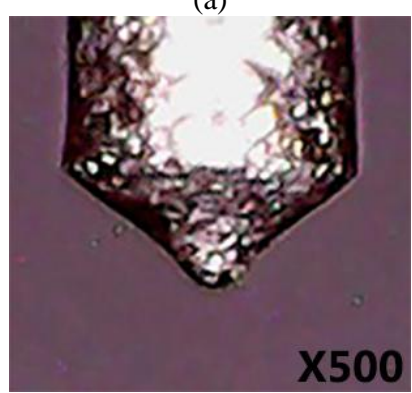

(e)

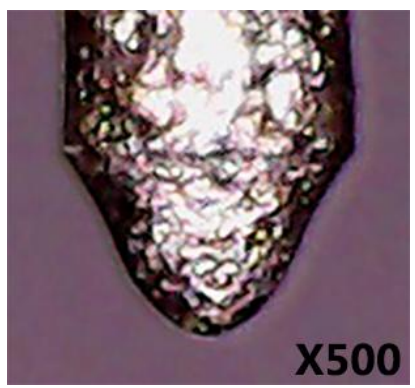

(b)

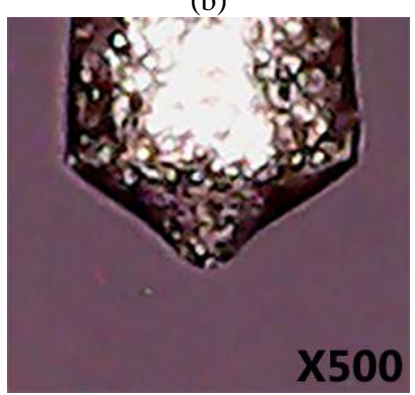

(f)

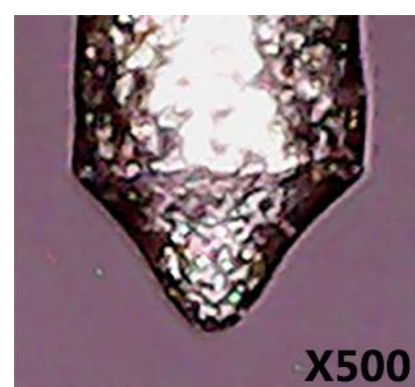

(c)

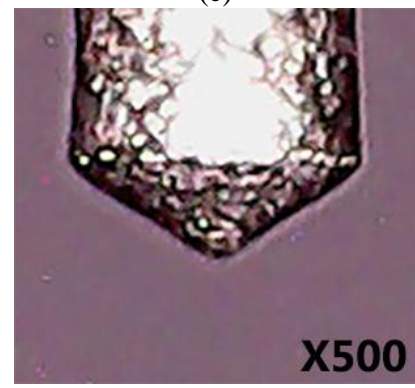

(g)

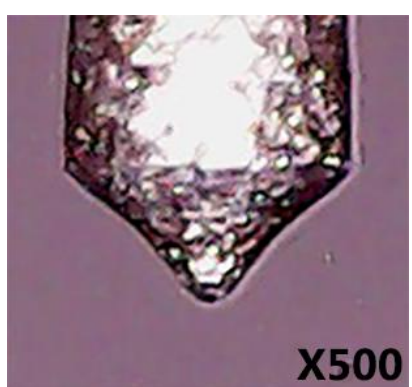

(d)

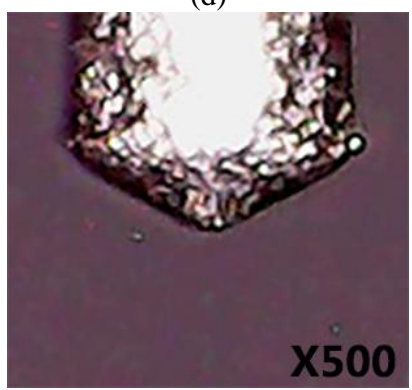

(h)

Figure 18 Change of tools shape in experiments of the fixed-length compensation with different milling lengths. (a) milling $171 \mu$ m; (b) milling $362 \mu \mathrm{m}$; (c) milling $546 \mu \mathrm{m}$; (d) milling $628 \mu \mathrm{m}$; (e) milling $794 \mu \mathrm{m}$; (f) milling $878 \mu \mathrm{m}$; (g) milling $1055 \mu \mathrm{m}$; (h) milling $1103 \mu \mathrm{m}$.

In Figures 17 (d) to (f), the conic shape of the tool forms, which is in picture-wise accordance with the corresponding experimental results shown in Figures 18 (d) to (f). It is of great interest to explore more details beyond previous study based on the geometric model done by Zhang et al. ${ }^{[12]}$. Notice that when the shape of the tool reaches stable as in Figures $17(\mathrm{~g})$ and $(\mathrm{h})$, the tip of the tool is not that sharp as the simulated results presented in Figures $7(\mathrm{~g})$ and $(\mathrm{h})$ by Zhang et al. ${ }^{[12]}$ using the geometric model. Comparing with corresponding experimental results, one can verify that this relatively flat and smooth tip obtained by the electric field simulation can better depict the electrode profile of real machining. Physically speaking, this breakthrough in the prediction of the tool shape was made by considering the corona discharging effects at tips using EM theory, which cannot be addressed by the geometric model. Again, in the electric field model, high sparking probability is retained at every tip of the tool due to the large curvatures till they are worn off in the long run, thus leading to smoothness on the profile.

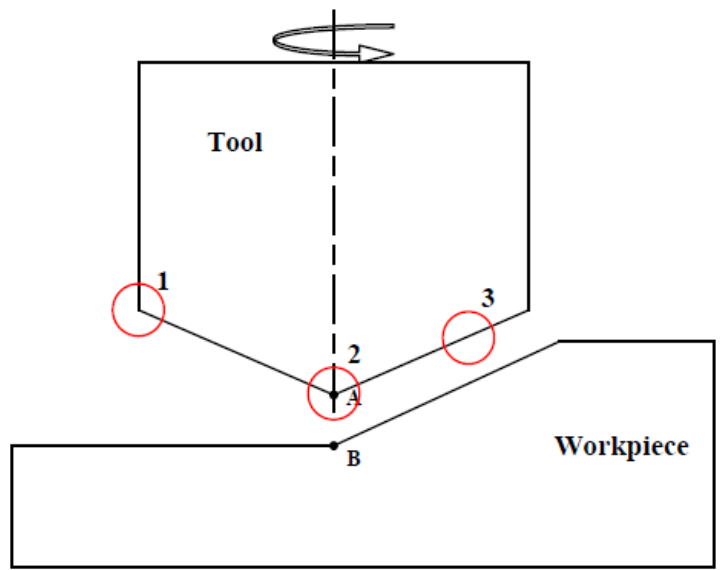

(a)

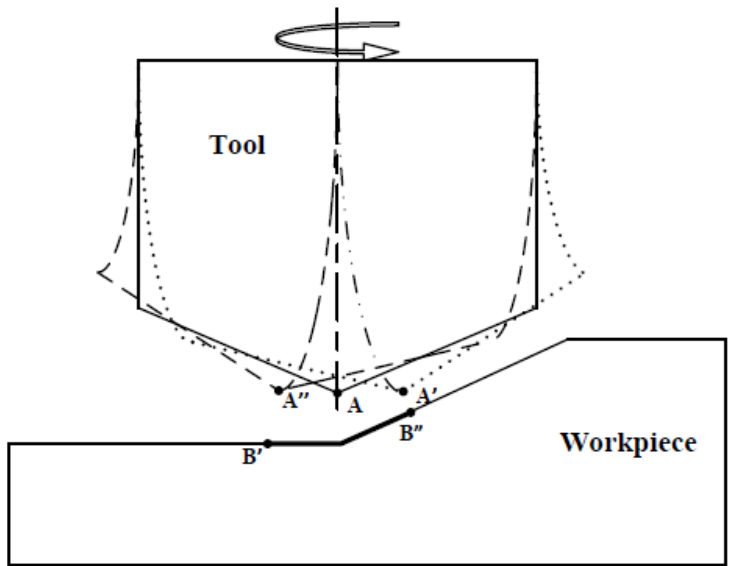

(b)

Figure 19 Schematic of coaxiality during rotation of tool. (a) Perfectly (axisymmetric) tool; (b) Real tool. Large tool wear at tips due to coaxiality in reality.

To investigate the performance of the electric field model in the conic angle prediction, a series of experiments were conducted to study conic angles varying with layer thickness. In Figure 20, when layer thickness reaches $125 \mu \mathrm{m}$, the simulated conic angle by the electric field model is 45.3 degrees, which matches with the experimental results with an error of $4.86 \%$, and the errors for large layer thickness in the electric field model (purple line) are significantly decreased in comparison to the geometric model (green line). Compared with the result of geometric model with the same parameters by Zhang et al. ${ }^{[12]}$, the proposed electric field model shows better accuracy in such predictions. 


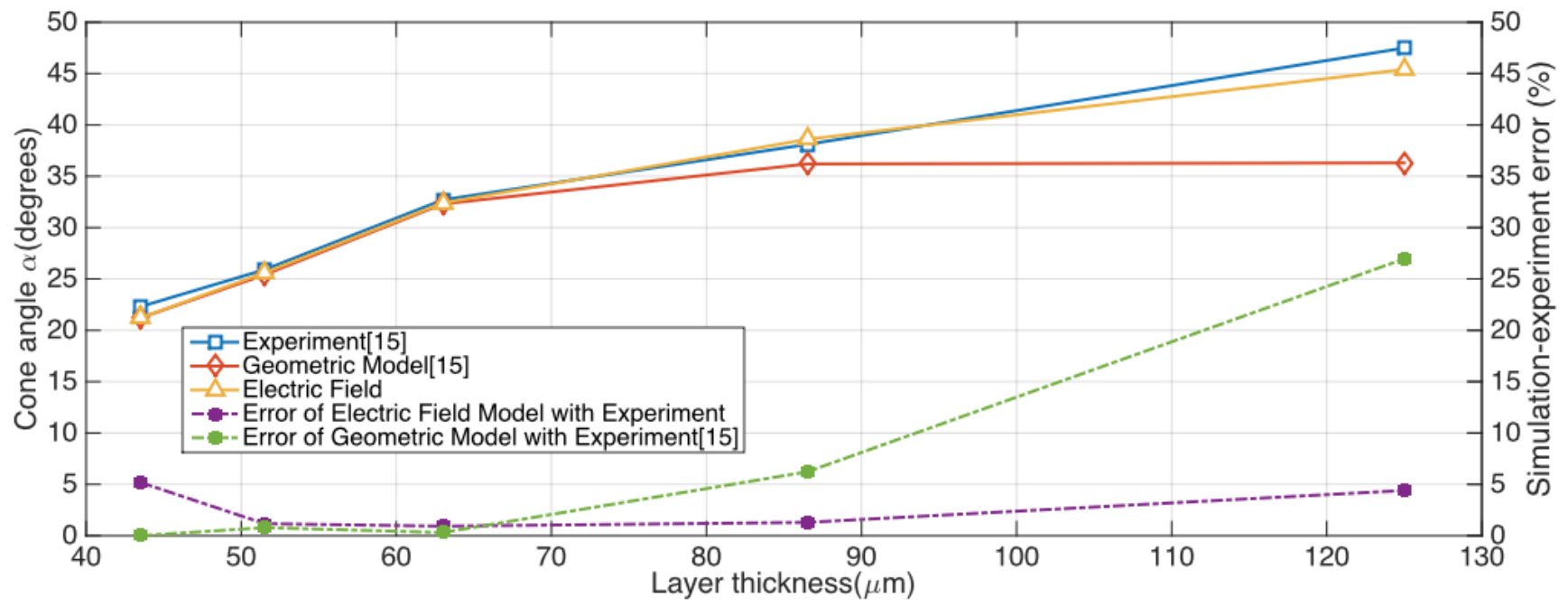

Figure 20 Conic angle varying with layer thickness for simulation and experiment results.

Further interpretations about the electric field model outperforming the geometric model in predicting the conic angle at large layer thickness are demonstrated here. The fix-length compensation features a tiny amount of axial compensation after horizontal milling by a relatively larger fixed distance. It means in the geometric model, the electrode feeds horizontally many more times than vertically, and this causes the shortest distance to occur on the periphery rather than on the end face. Because sparking is triggered depending on the shortest distance between electrodes, the wear ratio of the periphery to the end face is expected to be high. As the wear ratio of the periphery to the tool end face determines the angle of the tool ${ }^{[12]}$, the tool thus becomes slim rapidly. When the radius of tool end is close to zero, part of the tool inserted into the workpiece will suddenly disappear, which leads to a dramatic decrease of the conic angle, especially when the layer thickness is large. However, in the electric field model, even though the gap between the tool and the workpiece is larger than that between the periphery and the workpiece, it is still the tool end which sparks and wears due to its large curvature. In this way, the wear ratio of the periphery to the tool end face will decrease, and thus the large conic angle in large layer thickness simulation is achieved.

\section{Conclusion}

A model of tool wear in EDM based on electromagnetic theory is presented in this paper. Different from the traditional geometric model, the sparking in this work is determined through the electric field rather than the gap distance between electrodes. The Gauss equation is solved numerically in each time step to find the intensity maximum exceeding the breakdown strength of the dielectric medium, at which point the sparking occurs. From physical perspective, the effects of corona discharging at sharp tips can be naturally incorporated into this sparking theory, and thus this electric model is capable of providing a more accurate prediction on tool wear.

To evaluate the accuracy of the method, two types of test are carried out. The first type is on the die sinking simulation. Through the numerical results, the larger sparking probability at the sharp tips is found by mapping the sparking frequency along the surface of the tool. This high sparking probability represents the corona discharging which accounts for the large tool wear at tips observed in experiments. The second set of test considers the milling simulation. A special milling process named fix-length compensation method is simulated using the developed model. With the help of this electric field model, the tool shape during different milling stages and the stable conic angle at large layer thickness can be precisely predicted. As it has been proved that the fix-length compensation milling cannot be fully simulated by previous geometric model, the electric field model is more well-defined in predicting complex EDM process than its counterpart.

Enhanced understanding of the corona discharging effect on EDM simulation is also achieved by comparing the electric field model with the geometric one. Generally speaking, it is the sparking frequency that attributes to the surface curvature in the electric field model rather than the edge density in the geometric model. As a result, when the tool end is generally smooth, the effect of corona discharging is not dominant, hence the geometric model can produce reasonable results. However, when complex curvatures feature the tool end, such as the conic, wedged or the dual-tip tools used in this study, the corona discharging effect becomes significant, and the electric model should be applied instead.

\section{Acknowledgment}

The project is supported by the National Science Foundation China (Grant no. 51205252). 


\section{References}

[1] K.H Ho and S.T Newman. State of the art electrical discharge machining (edm). International Journal of Machine Tools and Manufacture, 13(10):1287-1300, 2003.

[2] Naotake Mohri, Masayuki Suzuki, Masanori Furuya, Nagao Saito, and Akira Kobayashi. Electrode wear process in electrical discharge machinings. CIRP Annals - Manufacturing Technology, 44(1):165-168, 1995.

[3] D.T Pham, S.S Dimov, S Bigot, A Ivanov, and K Popov. Micro-edm recent developments and research issues. Journal of Materials Processing Technology, 149(6):50-57, 2004.

[4] Z. Y. Yu, J. Kazak, and K. P. Rajurkar. Modelling and simulation of micro edm process. CIRP Annals - Manufacturing Technology, 52:143-146, 2003.

[5] D.T Pham, S.S Dimov, S Bigot, A Ivanov, and K Popov. An investigation of tube and rod electrode wear in micro edm drilling. The International Journal of Advanced Manufacturing Technology, 33(5):103-109, 2007.

[6] K. P. Somashekhar, S. Panda, J. Mathew, and N. Ramachandran. Numerical simulation of micro-edm model with multispark. International Journal of Advanced Manufacturing Technology, 83:90, 2015.

[7] G. Bissacco, J. Valentincic, H. N. Hansen, and B. D. Wiwe. Towards the effective tool wear control in micro-edm milling. International Advanced Manufacturing Technology, 3:3-9, 2010.

[8] D. T Pham, A Ivanov, S Bigot, K Popov, and S. Dimov. A study of micro-electro discharge machining electrode wear. Proceedings of the Institution of Mechanical Engineers, 221(5):605-612, 2007.

[9] Young Hun Jeong and Byung-Kwon Min. Geometry prediction of edm-drilled holes and tool electrode shapes of micro-edm process using simulation. International Journal of Machine Tools and Manufacture, 47(12):1817-1826, 2007.

[10] Segon Heo et al. Virtual edm simulator: three-dimensional geometric simulation of micro-edm milling processes. International Journal of Machine Tools and Manufacture, 49(12):1029-1034, 2009.

[11] M. Kunieda and M. Kivohara. Simulation of die-sinking EDM by discharge location searching algorithm. International Journal of Electrical Machining, 3:79- 85, 1998.

[12] Lenan Zhang, Jianyi Du, Xiaoshun Zhuang, ZhiliangWang, and Jingyu Pei. Geometric prediction of conic tool in micro-edm milling with fix-length compensation using simulation. International Journal of Machine Tools and Manufacture, 89(2):86-94, 2015.

[13] Z. Y. Yu, T. Masuzawa, and M. Fujino. Micro-edm for three-dimensional cavities - development of uniform wear method. CIRP Annals - Manufacturing Technology, 47:169-172, 1998.

[14] E. Aligiri, S. H. Yeo, and P. C. Tan. A new tool wear compensation method based on real-time estimation of material removal volume in micro-edm. Journal of Materials Processing Technology, 210:2292-2303, 2010.

[15] Fuzhu Han, Yuji Yamada, Taichi Kawakami, and Masanori Kunieda. Experimental attempts of sub-micrometer order size machining using micro-edm. Precision Engineering, 30:123-131, 2006.

[16] P. Govindan and Suhas S. Joshi. Experimental characterization of material removal in dry electrical discharge drilling. International Journal of Machine Tools and Manufacture, 50:431-443, 2010.

[17] Xiaodong Yang, Jianwen Guo, Xiaofei Chen, and Masanori Kunieda. Molecular dynamics simulation of the material removal mechanism in micro-edm. Precision Engineering, 35:51-57, 2011.

[18] Jianwen Guo, Guojun Zhang, Yu Huang, Wuyi Ming, Min Liu, and Hao Huang. Investigation of the removing process of cathode material in micro-edm using an atomistic-continuum model. Applied Surface Science, 315:323-336, 2014.

[19] S. N. Joshi and S. S. Pande. Development of an intelligent process model for edm. International Journal Advanced Manufacturing Technology, 45:300-317, 2009.

[20] Jingyu Pei et al. Arithmetic and experimental study of fix-length compensation based on conical bottom shape of electrode in micro-edm. ASME 2013 International Mechanical Engineering Congress and Exposition, 2013. 